\title{
TWO-DIMENSIONAL GRAVITATION AND SINE-GORDON-SOLITONS
}

\author{
Bernd Stoetzel * \\ Institute for Theoretical Physics \\ Department of Physics, University of Alberta \\ Edmonton, T6G 2J1 Alberta, Canada
}

August 21, 2018

\begin{abstract}
Some aspects of two-dimensional gravity coupled to matter fields, especially to the Sine-Gordon-model are examined. General properties and boundary conditions of possible soliton-solutions are considered. Analytic soliton-solutions are discovered and the structure of the induced space-time geometry is discussed. These solutions have interesting features and may serve as a starting point for further investigations.
\end{abstract}

\section{Introduction}

Since the first purely pedagogical study of two-dimensional gravitation [1] there has been considerable interest in that topic for the last years [2 - 8]. Apart from being an interesting subject in itself, two-dimensional models proved to be very useful tools for studying effects, whose description is rather complicated in realistic fourdimensional gravitation. Examples are gravitational collapse [3], black holes [4], cosmologies [5] and quantum effects [6].

Unfortunately, it is not possible to use the Einstein-equations in two dimensions, because the Einstein-action is a topological invariant and therefore, the Einsteintensor is identically zero. Several suggestions were made to circumvent this problem: Jackiw [7] and Teitelboim [8] proposed a model which they essentially got by dimensional reduction of the three-dimensional Einstein-action supplemented with a cosmological constant and an auxiliary field. Mann, et al. [9] extended this model by giving dynamical content to the auxiliary field. Another approach was pursued by Callan, et al. [10] who considered a string-theory inspired action. With the help

\footnotetext{
*Supported by Deutsche Forschungsgemeinschaft. E-mail: Stoetzel@phys.ualberta.ca
} 
of a new parameter it is possible to unite various actions into one, as was shown by Lemos and Sá [11]. These different models will be briefly reviewed in section two.

Much of the analysis of two-dimensional models was concerned with vacuumsolutions (especially black holes [4), but it is of course possible to include also different kinds of matter-couplings to study the mutual influence of matter and gravity. A very useful example is the above mentioned model of Callan, et al. [10], coupled to massless scalar-fields, because it is exactly soluble on the classical level. Also, quantum effects, like Hawking-radiation, can be analysed. It is therefore interesting to see, if any other matter-field models allow analytic classical solutions as a starting point for considerations of quantum effects.

As is well-known for many years, there is such a model in flat space-time, namely the Sine-Gordon-model, whose nonlinear interaction potential provides classical particle-like kink-solutions [12]. It proved to be very useful for studying quantization techniques of nonlinear baryon-models like the Skyrme-model [13]. Skyrmions coupled to gravity were analysed recently by Heusler, et al. [14]. Therefore, it is suggestive to study the gravitational Sine-Gordon-model, which is the main purpose of this paper.

In section three some features of the Sine-Gordon-model in flat space-time and their generalization to curved space-time are discussed. General aspects of the gravitational Sine-Gordon-model and its equations of motion are considered in section four and furthermore, the boundary conditions for possible soliton-solutions are ex-

amined. Some speculations on simple modifications of the Sine-Gordon-model are presented. In section five various types of analytic solutions are listed and the structure of the kink and of the space-time for these different solutions is discussed

\section{Two-dimensional models for gravity}

It is well-known that the Einstein-tensor $G_{\mu \nu}$ vanishes identically in two dimensions. Therefore, it is not possible to use the usual Einstein-equations

$$
G_{\mu \nu}=8 \pi G T_{\mu \nu}
$$

As a consequence, one has to think about suitable alternatives. Jackiw [7] and Teitelboim [8] suggested the so-called constant-curvature-model

$$
R+\Lambda=0
$$

In four dimensions this is simply the trace of the vacuum Einstein-equations with cosmological constant. It is useful to have an action whose variation gives the equations of motion. In four dimensions such an action is (for the vacuum-equations)

$$
I_{E}=\int d^{4} x \sqrt{-g} R
$$


It is also possible to construct a two-dimensional action leading to (2), but involving an auxiliary scalar-field:

$$
I=\int d^{2} x N \sqrt{-g}(R+\Lambda)
$$

Varying $N$ immediately gives (2), whereas the variation with respect to the metric yields an equation for the field $N$. This action can also be understood as a dimensionally reduced form of the three-dimensional Einstein-action. It is then easy to include matter-interaction in this model by adding a matter-term to the action:

$$
I_{J T}=\frac{1}{\kappa} \int d^{2} x N \sqrt{-g}\left(R+\Lambda+\kappa \mathcal{L}_{M}\right)
$$

where $\mathcal{L}_{M}$ is the matter-lagrangian and $\kappa$ a coupling constant. In this case the variational equations are

$$
\begin{aligned}
N_{; \alpha \beta}-g_{\alpha \beta} N_{; \mu}^{; \mu} & =-\frac{1}{2} \kappa N T_{\alpha \beta} \\
R+\Lambda & =-\kappa \mathcal{L}_{M}
\end{aligned}
$$

with the energy-momentum-tensor defined as

$$
T_{\alpha \beta}=g_{\alpha \beta} \mathcal{L}_{M}-2 \frac{\delta \mathcal{L}_{M}}{\delta g^{\alpha \beta}}
$$

One important aspect of this model is that the energy-momentum-tensor is not covariantly conserved.

A natural extension of the Jackiw-Teitelboim-model, which yields a conserved energy-momentum was suggested by Mann, et al. [9] who gave dynamical content to the auxiliary field. The action is then (omitting the cosmological constant)

$$
I_{M}=\frac{1}{\kappa} \int d^{2} x \sqrt{-g}\left(-\frac{1}{2} \partial^{\mu} \psi \partial_{\mu} \psi+\psi R+\kappa \mathcal{L}_{M}\right)
$$

Instead of (6) the equations of motion now read

$$
\begin{gathered}
\psi_{; \mu}^{; \mu}=-R \\
\psi_{; \mu} \psi_{; \nu}+2 \psi_{; \mu \nu}-\frac{1}{2} g_{\mu \nu}\left(\psi^{; \alpha} \psi_{; \alpha}+4 \psi_{; \alpha}^{; \alpha}\right)=-\kappa T_{\mu \nu}
\end{gathered}
$$

Taking the trace of (10) yields, together with (9),

$$
\psi_{; \mu}^{; \mu}=\frac{1}{2} \kappa T=-R
$$

This equation is in direct analogy to the trace of the four-dimensional Einsteinequation $R \sim T$.

A rather different approach was pursued by Callan, et al. [10 who used a stringtheory inspired action to analyse black hole evaporation by Hawking radiation:

$$
I_{S T}=\frac{1}{\kappa} \int d^{2} x \sqrt{-g}\left\{e^{-2 \varphi}\left[R+4 \partial^{\mu} \varphi \partial_{\mu} \varphi\right]+\kappa \mathcal{L}_{M}\right\}
$$


Lemos and Sá [11] recently proposed a model which unites all these different actions into one by introducing a constant parameter:

$$
I_{B D}=\frac{1}{\kappa} \int d^{2} x \sqrt{-g}\left\{e^{-2 \varphi}\left[R-4 \omega \partial^{\mu} \varphi \partial_{\mu} \varphi\right]+\kappa \mathcal{L}_{M}\right\}
$$

This is a Brans-Dicke type action in two dimensions [15]. For $\omega=-1$ one obtains (12) and for $\omega=0$ one rediscovers the Jackiw-Teitelboim model (5) (apart from a slightly different matter coupling). The model of Mann, et al. is covered by the limit $\omega \rightarrow \infty$ 16. The variational equations for this general action is

$$
\begin{gathered}
R=4 \omega\left(\varphi_{; \mu}^{; \mu}-\varphi^{; \mu} \varphi_{; \mu}\right) \\
\varphi_{; \mu \nu}-g_{\mu \nu} \varphi_{; \alpha}^{; \alpha}-2(\omega+1) \varphi_{; \mu} \varphi_{; \nu}+g_{\mu \nu}(\omega+2) \varphi^{; \alpha} \varphi_{; \alpha}=\frac{1}{4} \kappa e^{2 \varphi} T_{\mu \nu}
\end{gathered}
$$

Which of these different models may be regarded as two-dimensional general relativity is more or less a matter of taste. In the following sections I prefer the model of Mann, et al. for several reasons. First, this model yields as one equation of motion the analogue of the trace of the Einstein-equations $R \sim T$. Second, it may be obtained as the limit $\omega \rightarrow \infty$ of the Brans-Dicke action (13) which, in four dimensions, actually gives the Einstein-model of general relativity 16]. Third, it can be considered as the $D \rightarrow 2$ limit of $D$-dimensional gravity, as was shown by Mann and Ross [17]. The fourth reason is rather pragmatic, but nonetheless important in the search for analytic solutions: the equations are not too complicated.

\section{Sine-Gordon-model}

Before considering the coupled system of gravity and Sine-Gordon-model, first, I would like to review some of the main features of the latter in flat space-time.

The Sine-Gordon-model is a two-dimensional model for a massless scalar-field $\phi$ with a sinus-type self-interaction, given by

$$
\mathcal{L}_{S G}=-\frac{1}{2} \partial^{\mu} \phi \partial_{\mu} \phi-U(\phi)
$$

with

$$
U(\phi)=2 m^{2} \sin ^{2} \frac{\phi}{2}=m^{2}(1-\cos \phi)
$$

This lagrangian was extensively studied as a tool for testing different soliton quantization methods, which was of great interest in the study of solitonic baryon-models, like the Skyrme-model [13]. Another subject of analysis appeared after the discovery of Coleman [18] that the Sine-Gordon-model (describing bosons) is equivalent in a certain sense to the two-dimensional Thirring-model (describing fermions). Therefore, the Sine-Gordon-model was very fruitful in the study of different aspects of

quantum field theory and there is some hope that this might also be true for the gravity-coupled Sine-Gordon-model. 
An especially interesting feature of the potential (17) is that it admits solitonsolutions. Consider the equation of motion for the lagrangian (16) in the static case

$$
\phi^{\prime \prime}=m^{2} \sin \phi
$$

which has the solution

$$
\phi=4 \arctan e^{ \pm m\left(x-x_{0}\right)}
$$

This solution connects two successive minima of the potential $U(\phi)$ or, in other words, two distinct vacua of the Sine-Gordon-model. Examining the energy-density for the solution (19) reveals another important feature of solitons, namely that its energy is bound and restricted to a certain area of space, which makes it possible to assign a mass and a size to the soliton. The energy-density is

$$
\mathcal{H}=\frac{1}{2}\left[\phi^{\prime 2}+2 U(\phi)\right]=\phi^{\prime 2}=\frac{4 m^{2}}{\cosh ^{2}\left[m\left(x-x_{0}\right)\right]}
$$

$\mathcal{H}$ has its maximum at $x=x_{0}$, which may therefore be regarded as the position of the soliton. In the following I take $x_{0}=0$. The mass of the soliton is

$$
M=\int_{-\infty}^{\infty} d x \mathcal{H}(x)=8 m
$$

The most important property of the solution (19) is that one can assign a conserved soliton-number to it, which may be defined as the spatial integral over the zeroth component of the obviously conserved current

$$
j^{\mu}=\frac{1}{2 \pi} \epsilon^{\mu \nu} \partial_{\nu} \phi
$$

Where $\epsilon^{\mu \nu}$ is the two-dimensional Levi-Civita-symbol with $\epsilon^{01}=1$. For (19), the soliton-number is

$$
K=\int_{-\infty}^{\infty} d x j^{0}=\frac{1}{2 \pi} \int_{-\infty}^{\infty} d x \phi^{\prime}= \pm 1
$$

Therefore, the solution with the +sign is called soliton and the one with the - sign antisoliton. Concerning the generalization of these concepts to curved space-time, the soliton-mass is not well defined, because of the usual problems in defining locally conserved energy in general relativity, whereas the notion of soliton-number, being a topological property of the solution, is the same in curved as well as in flat spacetime. The reason for this is that the current (22) stays to be locally conserved:

$$
\begin{aligned}
j_{; \mu}^{\mu} & =\frac{1}{2 \pi} \epsilon^{\mu \nu} \phi_{; \nu \mu}=0 \\
& =\frac{1}{\sqrt{-g}}\left(\sqrt{-g} j^{\mu}\right)_{{ }_{\mu}}
\end{aligned}
$$


Consequently, one can define the soliton-number in curved space as

$$
K=\int_{-\infty}^{\infty} d x \sqrt{-g} j^{0}
$$

\section{Gravitational Sine-Gordon-model}

For the reasons discussed in section 2 consider now the following action describing a gravitational Sine-Gordon-model:

$$
I=\frac{1}{\kappa} \int d^{2} x \sqrt{-g}\left[-\frac{1}{2} \partial^{\mu} \psi \partial_{\mu} \psi+\psi(R+\Lambda)+\kappa \mathcal{L}_{S G}(\phi)\right]
$$

where $\mathcal{L}_{S G}$ is the lagrangian (16), $\psi$ is the necessary auxiliary field as introduced by Mann, et al., $R$ is the usually defined scalar curvature, $g$ the determinant of the metric tensor $g_{\mu \nu}$ ( I use the signature $(-,+,+,+)$ ), $\Lambda$ is a cosmological constant and $\kappa$ is the coupling-constant which is required to be positive. Otherwise, the action would rather describe an antigravitational Sine-Gordon-model. An example for a solution with negative $\kappa$ is given in appendix A. It should be noted that the matter-gravity coupling in (26) is not conformally invariant. Such type of a coupling is also considered by Ambjørn and Ghoroku [19]. The variation of (26) with respect to $\psi$ and $g_{\mu \nu}$ gives equation (9) and (10) of section 2 , which can also be written in the form (11)

$$
R=-\frac{1}{2} \kappa T-\Lambda
$$

plus equation (10) for the auxiliary field $\psi$, which is regarded as a consistency equation without any physical meaning. $T$ in (27) is the trace of the energy-momentumtensor defined in $(7)$ :

$$
\begin{aligned}
T_{\mu \nu} & =g_{\mu \nu} \mathcal{L}_{S G}-2 \frac{\delta \mathcal{L}_{S G}}{\delta g^{\mu \nu}} \\
& =\partial_{\mu} \phi \partial_{\nu} \phi-\frac{1}{2} g_{\mu \nu}\left[\partial^{\alpha} \phi \partial_{\alpha} \phi+2 U(\phi)\right]
\end{aligned}
$$

The trace is therefore

$$
T=-2 U(\phi)
$$

The scalar-field potential is the source for the metric. One needs of course also an equation for the scalar-field itself. Variation of the action with respect to $\phi$ yields

$$
\phi_{; \mu}^{; \mu}=U^{\prime}(\phi)
$$

where the prime denotes derivation with respect to $\phi$. With the help of this equation it is easy to show that the energy-momentum is covariantly conserved:

$$
\begin{aligned}
T_{; \nu}^{\mu \nu} & =\left(\phi^{; \mu} \phi^{; \nu}\right)_{; \nu}-\frac{1}{2} g^{\mu \nu}\left[\left(\phi^{; \alpha} \phi_{; \alpha}\right)_{; \nu}+2 U^{\prime}(\phi) \phi_{; \nu}\right] \\
& =\phi_{; \nu} \phi^{; \mu \nu}-\phi_{; \nu} \phi^{; \nu \mu}+\phi^{; \mu} \phi_{; \nu}^{; \nu}-\phi^{; \mu} U^{\prime}(\phi) \\
& =0
\end{aligned}
$$


Two-dimensional gravity has only one degree of freedom, which means that the metric is completely characterized by just one function. For the present purpose it proves to be best to consider a metric of the following form:

$$
d s^{2}=-e^{2 \varphi} d t^{2}+d x^{2}
$$

Each two-dimensional metric can be brought to that form by a suitable coordinate transformation. I am interested in static solutions, which means $\phi=\phi(x)$ and $\varphi=\varphi(x)$. In that case the scalar curvature for the metric (32) becomes

$$
R=-2\left(\varphi^{\prime \prime}+\varphi^{2}\right)
$$

where the prime denotes derivation with respect to $x$. Furthermore, the second covariant derivative of a scalar-field is

$$
\begin{aligned}
\phi_{; \mu \nu} & =\phi^{\prime \prime} \delta_{\mu}^{x} \delta_{\nu}^{x}-\varphi^{\prime} \phi^{\prime} e^{2 \varphi} \delta_{\mu}^{t} \delta_{\nu}^{t} \\
\phi_{; \mu}^{; \mu} & =\phi^{\prime \prime}+\varphi^{\prime} \phi^{\prime}
\end{aligned}
$$

Accordingly, one has to consider the following static equations:

$$
\begin{aligned}
\phi^{\prime \prime}+\theta \phi^{\prime} & =U^{\prime}(\phi) \\
\theta^{\prime}+\theta^{2} & =-\frac{1}{2} \kappa U(\phi)+\frac{1}{2} \Lambda
\end{aligned}
$$

with the function $\theta \equiv \varphi^{\prime}$. For given potential $U(\phi)$ these are coupled and nonlinear, but ordinary differential equations which may, in principle, be solved for given boundary conditions. A general solution is possible in the easiest case of a massless scalar-field $U(\phi)=0$. It is especially interesting that there exists a soliton-solution in that case, which can be interpreted as a gravitationally bound kink. This special solution is discussed in appendix B. Consider now nonlinear potentials like the SineGordon-potential. What are suitable boundary conditions for a soliton-solution? As can be seen from the example of the flat-space Sine-Gordon-model a soliton connects two minima of the potential $U(\phi)$ and is monotonically rising (19):

$$
\begin{array}{rll}
\phi_{-} \leq \phi \leq \phi_{+} & ; & \phi^{\prime} \geq 0 \\
\phi^{\prime}\left(\phi_{ \pm}\right)=0 & ; \quad & U\left(\phi_{ \pm}\right)=U_{ \pm}
\end{array}
$$

where $\phi_{ \pm}=\phi(x \rightarrow \pm \infty)$ and $U^{\prime}\left(\phi_{ \pm}\right)=0$.

To analyse possible solutions of the equations (35) and (36), with the boundary conditions (37), it is best to decouple the equations. This is achieved in the following way. Derivation of (36) with respect to $x$ and insertion of (35) yields

$$
\begin{aligned}
\theta^{\prime \prime}+2 \theta \theta^{\prime} & =-\frac{1}{2} \kappa U^{\prime}(\phi) \phi^{\prime}=-\frac{1}{2} \kappa\left(\phi^{\prime \prime}+\theta \phi^{\prime}\right) \phi^{\prime} \\
& =-\frac{1}{4} \kappa\left[\left(\phi^{\prime 2}\right)^{\prime}+2 \theta \phi^{\prime 2}\right]
\end{aligned}
$$

A special solution of this equation obviously is

$$
\theta^{\prime}=-\frac{1}{4} \kappa \phi^{\prime 2}
$$


If one reinserts this relation into (36) one obtains the function $\theta$ in dependence of the function $\phi$ :

$$
\theta^{2}=\frac{1}{4} \kappa\left[\phi^{\prime 2}-2 U(\phi)\right]+\frac{1}{2} \Lambda
$$

Equation (35), multiplied with $\phi^{\prime}$, then becomes an equation for $\phi$ alone:

$$
\frac{1}{2}\left(\phi^{\prime 2}\right)^{\prime} \pm \frac{\sqrt{\kappa}}{2} \phi^{\prime 2} \sqrt{\phi^{\prime 2}-2 U(\phi)+\frac{2 \Lambda}{\kappa}}=[U(\phi)]^{\prime}
$$

where all primes now denote derivation with respect to $x$. This can also be written as

$$
{\sqrt{\phi^{\prime 2}-2 U(\phi)+\lambda}}^{\prime}= \pm \frac{\sqrt{\kappa}}{2} \phi^{\prime 2}
$$

with the rescaled cosmological constant $\lambda$. This seems to be rather complicated, but may be simplified in the following way. Assume, there is a solution $\phi=\phi(x)$ and $\phi^{\prime}=\phi^{\prime}(x)$. One may now invert the equation for $\phi$ and insert it in the one for $\phi^{\prime}$ to get $\phi^{\prime}$ as a function of $\phi$. Call this function $F^{\prime}(\phi)$. Thus,

$$
F^{\prime}(\phi) \equiv \frac{d F(\phi)}{d \phi}=\phi^{\prime}(x) \equiv \frac{d \phi(x)}{d x}
$$

and

$$
[F(\phi)]^{\prime} \equiv \frac{d F(\phi)}{d x}=F^{\prime}(\phi) \phi^{\prime}(x)=\phi^{\prime 2}
$$

Equation (42) can then be written as

$$
{\sqrt{F^{\prime 2}-2 U+\lambda}}^{\prime}= \pm \frac{\sqrt{\kappa}}{2}[F(\phi)]^{\prime}
$$

or, after one integration,

$$
\sqrt{F^{\prime 2}-2 U+\lambda}= \pm \frac{\sqrt{\kappa}}{2} F
$$

The integration constant may be set to zero, because $F$ is defined only up to a constant. Squaring the whole equation then yields a first order differential equation for the function $F(\phi)$ :

$$
F^{2}-\frac{1}{4} \kappa F^{2}=2 U-\lambda
$$

It is now possible to translate (37) into conditions for $F$ :

$$
F^{\prime}(\phi) \geq 0 ; \quad F^{\prime}\left(\phi_{ \pm}\right)=0
$$

From (47) one further may infer

$$
F^{2}\left(\phi_{ \pm}\right)=-\frac{4}{\kappa}\left(2 U_{ \pm}-\lambda\right)
$$


This means that solutions for positive $\kappa$ are possible if $2 U_{ \pm}-\lambda \leq 0$. $F(\phi)$ is a monotonically rising function as well as $\phi(x)$, thus, for the Sine-Gordon-model $\left(U_{ \pm}=0\right)$

$$
F\left(\phi_{ \pm}\right)= \pm \frac{2}{\sqrt{\kappa}} \sqrt{\lambda}
$$

A soliton-solution of equation (42) is therefore possible for a positive cosmological constant. What can be said about the metric function $\theta$ ? $\theta$ is determined by equation (40) and the boundary conditions of $\theta$ compatible with that equation are

$$
\theta^{2}(x \rightarrow \pm \infty)=-\frac{1}{4} \kappa\left[2 U_{ \pm}-\lambda\right]=\frac{1}{4} \kappa \lambda
$$

where the last equality holds for the Sine-Gordon-model. Solutions with vanishing cosmological constant are possible if at least one of the two constants $U_{ \pm}$is negative. An example is the following slightly modified Sine-Gordon-potential:

$$
U(\phi)=2 m^{2} \sin ^{2} \frac{\phi}{2}\left[1-\alpha \sin ^{2} \frac{\phi}{2}\right]
$$

Successive minima of this potential are

$$
U_{-}=0 ; \quad U_{+}=2 m^{2}(1-\alpha)
$$

and solutions are possible for $\alpha>1$. In the previous discussion I used the condition $\phi^{\prime}=0$, which is true only if $\phi(x)$ is defined on the whole real line $\Re$. Nevertheless, there may also be soliton-solutions having a compact domain $I=\left[x_{-}, x_{+}\right]$. This is possible if there are coordinate singularities at $x_{ \pm}$. In such a case (49) is replaced by

$$
F^{2}\left(\phi_{ \pm}\right)=-\frac{4}{\kappa}\left(2 U_{ \pm}-\lambda-\frac{1}{4} \kappa \phi_{ \pm}^{\prime 2}\right)
$$

where $\phi_{ \pm}^{\prime}=\phi^{\prime}\left(\phi_{ \pm}\right)$, and nothing can be said about the cosmological constant anymore. On the other hand, (40) shows that $\phi_{ \pm}^{\prime}$ has to become infinite, to ensure the coordinate singularities. For that reason there are no soliton-solutions with compact domain.

\section{$5 \quad$ Analytic solutions}

Although equation (47) looks rather simple, there is no standard procedure for solving it and I was not able to determine a general solution. The only possibility to find at least some special solutions which may be interpreted as solitons is by trial and error. Consider first the pure Sine-Gordon-model:

$$
F^{\prime 2}-\frac{1}{4} \kappa F^{2}=4 m^{2} \sin ^{2} \frac{\phi}{2}-\lambda
$$

The simplest possible ansatz subject to the conditions (48) is

$$
\begin{aligned}
& F^{\prime}(\phi)=A \sin \frac{\phi}{2} \\
& F(\phi)=F_{0}-2 A \cos \frac{\phi}{2}
\end{aligned}
$$


Insertion in (55) yields

$$
-\frac{1}{4} \kappa\left(F_{0}^{2}+4 A^{2}\right)+\kappa A F_{0} \cos \frac{\phi}{2}+A^{2}(1+\kappa) \sin ^{2} \frac{\phi}{2}=-\lambda+4 m^{2} \sin ^{2} \frac{\phi}{2}
$$

This equation determines the constants:

$$
F_{0}=0 ; \begin{array}{r}
-\kappa A^{2}=-\lambda \\
(1+\kappa) A^{2}=4 m^{2}
\end{array}
$$

For given Sine-Gordon-parameter $m$ and cosmological constant $\lambda$, one finds a solution

$$
F(\phi)= \pm 2 \sqrt{4 m^{2}-\lambda} \cos \frac{\phi}{2}
$$

but which is a solution only for the special value of the coupling-constant

$$
\kappa=\frac{\lambda}{4 m^{2}-\lambda}
$$

This is consistent with the requirement discussed in the previous chapter that a positive $\lambda$ is needed to get a solution for positive $\kappa$. In this special case here one finds an even stronger requirement, namely

$$
0<\lambda<4 m^{2}
$$

The scalar-field is now determined by (43):

$$
\phi^{\prime}(x)= \pm \sqrt{4 m^{2}-\lambda} \sin \frac{\phi}{2}
$$

which may be integrated at once to give

$$
\phi(x)=4 \arctan e^{ \pm M\left(x-x_{0}\right)}
$$

with the soliton mass-parameter

$$
M=\frac{1}{2} \sqrt{4 m^{2}-\lambda}
$$

This has the same form as the flat space kink and antikink solution, but with a different mass-parameter. One gets the original mass back for vanishing cosmological constant. As in flat space, $x_{0}$ may be interpreted as the center of the soliton and is set to zero in the rest of the paper. Also, I concentrate on the kink solution. The discussion for the antikink is of course completely analogous. Consider now the metric generated by the soliton. The metric function is given by (39):

$$
\begin{aligned}
\theta^{\prime} & =-\frac{1}{4} \kappa \phi^{\prime 2}=-\kappa M^{2} \sin ^{2} \frac{\phi}{2} \\
& =-\frac{\kappa M^{2}}{\cosh ^{2}(M x)}=-\frac{\lambda}{4 \cosh ^{2}(M x)}
\end{aligned}
$$


and by integration

$$
\varphi^{\prime}=\theta=-\frac{\lambda}{4 M} \tanh (M x)+\theta_{0}
$$

The integration-constant $\theta_{0}$ is determined by insertion in the original equations $(35$, 36 ), requiring $\theta_{0}=0$. One further integration then yields

$$
\varphi(x)=-\frac{\lambda}{4 M^{2}} \ln \cosh (M x)=-\kappa \ln \cosh (M x)
$$

Therefore, the soliton induced metric becomes

$$
d s^{2}=-\cosh ^{-2 \kappa}(M x) d t^{2}+d x^{2}
$$

This metric is similar to the different metrics analysed by Lemos and Sá [1] for general two-dimensional dilaton gravity. It is different, because it does not describe black holes, but the global structure of the space-time, however, is unexpectedly interesting and will be discussed below.

There is one point left to complete the examination of the equations of motion, namely to check if the consistency equation (10) for the auxiliary field $\psi$ has a solution. It is straightforward to show that this is actually the case with $\psi=2 \varphi$.

Before analysing the above solution, consider first the modified Sine-Gordonpotential (52) with vanishing cosmological constant. The same procedure as in the former case of the pure Sine-Gordon-model gives now a kink solution of the form

$$
\phi(x)=2 \arctan e^{m x}
$$

and the relation between the model-parameter $\alpha$ and the coupling-constant $\kappa$ is simply

$$
\kappa=4(\alpha-1)
$$

which again is in accordance with the in section 4 mentioned fact that $\alpha$ has to be greater than 1 to make solutions for positive $\kappa$ possible. The metric for the kink (69) is now

$$
d s^{2}=-\left(1+e^{2 m x}\right)^{-\frac{\kappa}{2}} d t^{2}+d x^{2}
$$

I turn now to a detailed discussion of the metric, starting with (68). The metric is obviously symmetric around the center of the kink and has coordinate singularities for $x \rightarrow \pm \infty$. From (33) one finds the scalar curvature

$$
R=\frac{2 \kappa m^{2}}{\cosh ^{2}(M x)}-\Lambda
$$

Therefore, the curvature is everywhere finite and no physical singularity arises. Nevertheless, the space-time covered by the coordinates $t$ and $x$ is not geodesically complete as may be seen from the geodesic equation

$$
g_{\alpha \beta} p^{\alpha} p^{\beta}+\mu^{2}=0
$$


which for the metric $(68)$ reduces to $\left(p_{0}=-E\right)$

$$
\begin{aligned}
\left(\frac{d x}{d \lambda}\right)^{2} & =E^{2} \cosh ^{2 \kappa}(M x)-\mu^{2} \\
& \approx E^{2}\left(\frac{1}{2} e^{ \pm M x}\right)^{2 \kappa}, \quad x \rightarrow \pm \infty
\end{aligned}
$$

The asymptotic behavior is

$$
\lambda \sim e^{\mp \kappa M x}, \quad x \rightarrow \pm \infty
$$

This means that the proper time lapse for a testparticle to travel from some point $x_{0}$ to $x \rightarrow \pm \infty$ is finite. To find an analytic extension of the solution (68) consider a Schwarzschild-like coordinate

$$
r=\int d x \cosh ^{-\kappa}(M x)
$$

The function $r(x)$ maps the domain $\Re$ of $x$ onto a compact interval $I$ whose boundary depends on $\kappa$. This provides the possibility to extend the metric analytically. To get an idea of the structure of the complete space-time examine two special choices of the coupling constant $\kappa$, namely $\kappa=1$ and $\kappa=2$. These values are the simplest ones, because the function $r(x)$ becomes explicitly invertible. For $\kappa=1$ one obtains

$$
r=\frac{2}{M} \arctan e^{M x}
$$

The image of this map is the interval $I=\left[0, \frac{\pi}{M}\right]$ and the metric has a Schwarzschildform in the coordinates $r$ and $t$ :

$$
d s^{2}=-\sin ^{2}(M r) d t^{2}+\frac{d r^{2}}{\sin ^{2}(M r)}
$$

By extending the domain of $r$ from the interval $I$ to $\Re$, one obtains a geodesically complete manifold. The coordinate-singularities are now located at the points $M r=$ $k \pi$ for $k=0, \pm 1, \ldots$. It is not possible to find a global coordinate system which is singularity-free (see appendix C). The complete manifold can be pictured as a "patchwork" of Penrose-diagrams of the form shown in figure 1.

In each of these space-time patches exists a soliton or antisoliton which, in the Schwarzschild-coordinate, has the form

$$
\phi(r)=2 M r
$$

Note that $M r$ is restricted for each soliton to one of the intervals $I_{k}=[k \pi,(k+1) \pi]$. By examining the geodesic equation analogous to (74)

$$
\left(\frac{d r}{d \lambda}\right)^{2}=E^{2}-\mu^{2} \sin ^{2}(M r)
$$




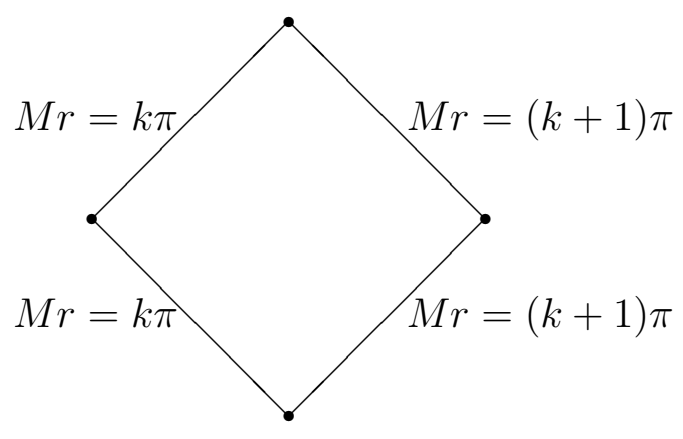

Figure 1: One patch of the Penrose-diagram for the Sine-Gordon-soliton with $\kappa=1$

one finds two types of geodesics. For $E>\mu$ the testparticle will travel from $r=-\infty$ to $r=\infty$ through infinitely many of the different patches, whereas for $E<\mu$ it will oscillate between two neighboring patches, hence, between neighboring solitons. Next consider the case $\kappa=2$. The Schwarzschild-coordinate is given by

$$
r=\frac{1}{M} \tanh (M x)
$$

The image of the map is now $I=\left[-\frac{1}{M}, \frac{1}{M}\right]$ and the metric has the form:

$$
d s^{2}=-\left(1-M^{2} r^{2}\right)^{2} d t^{2}+\frac{d r^{2}}{\left(1-M^{2} r^{2}\right)^{2}}
$$

The coordinate-singularities are located at $M r= \pm 1$. Other singularities arise at $r \rightarrow \pm \infty$ and these are now physical singularities as can be seen from the curvature scalar

$$
R=2 \kappa m^{2}\left(1-M^{2} r^{2}\right)-\Lambda
$$

Again it is not possible to find a globally singularity-free coordinate system. The Penrose-diagram for the extended space-time has the structure shown in figure 2. The soliton in this case has the form:

$$
\phi(r)=4 \arctan \sqrt{\frac{1+M r}{1-M r}}
$$

and is defined only in the regions corresponding to $-1 \leq M r \leq 1$. Possible geodesics are oscillations around $M r=0$ for $E>\mu$ and around $M r= \pm 1$ for $E<\mu$. The physical singularities at $r \rightarrow \pm \infty$ are unreachable for massive particles. Nevertheless, they are observable, because null geodesics may reach from $r=-\infty$ to $r=\infty$. In this sense the singularities in this space-time are naked. There is no horizon hiding them.

In the remaining part of this section I will discuss the metric (71), induced by the soliton (69) of the modified Sine-Gordon-potential (52) with vanishing cosmological constant. 


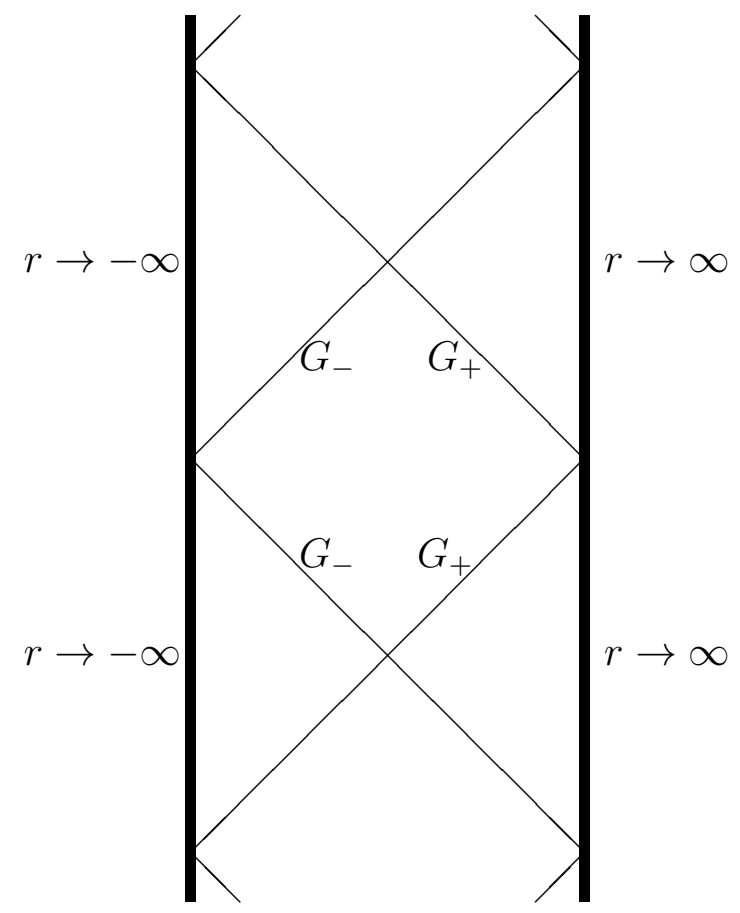

Figure 2: Penrose-diagram for the Sine-Gordon-soliton with $\kappa=1\left(G_{ \pm}\right.$denote lines with $M r= \pm 1$; bold lines represent physical singularities)

Calculating the kink-number (25) yields in this case

$$
K=\frac{1}{2 \pi} \int_{-\infty}^{\infty} d x \phi^{\prime}=\frac{1}{2 \pi}[\phi(\infty)-\phi(-\infty)]=\frac{1}{2}
$$

and one might expect an extension of the space-time which completes also the kink. The same conclusion may be drawn from the fact that the space-time covered by $t$ and $x$ is geodesically incomplete for $x \rightarrow \infty$, because the proper time lapse for testparticles approaching this limit is finite for the same reason as in the previous case. Also, in this limit the metric (71) is singular, but the space-time is not. The scalar curvature is finite at this end of the real line:

$$
R=\frac{\kappa m^{2}}{2 \cosh ^{2}(M x)}\left(1-\frac{\kappa}{4} e^{2 m x}\right)=-\frac{\kappa^{2} m^{2}}{2} ; \quad x \rightarrow \infty
$$

On the other side of the real line, $x \rightarrow-\infty$, the curvature vanishes, $R=0$, and the space-time is asymptotically flat.

To find an analytic extension switch again to a Schwarzschild-coordinate defined by

$$
r=\int d x\left(1+e^{2 m x}\right)^{-\frac{\kappa}{4}}
$$


The image of the function $r(x)$ is now the half-open interval $I=(-\infty, 0]$ and an extension into the region $r>0$ is possible. Again the specific structure of the complete space-time depends on the value of $\kappa$ and as examples I consider in the following the cases $\kappa=2$ and $\kappa=4$.

For $\kappa=2$ the coordinate transformation (87) is explicitly

$$
r(x)=\frac{1}{2 m} \ln \frac{\sqrt{1+e^{2 m x}}-1}{\sqrt{1+e^{2 m x}}+1}
$$

which yields the metric

$$
d s^{2}=-\tanh ^{2}(m r) d t^{2}+\operatorname{coth}^{2}(m r) d r^{2}
$$

Now the space-time is asymptotically flat at both sides of the real line and the structure of the space-time is analogous to an extreme Kerr-geometry given by the Penrose-diagram of figure 3 [20].

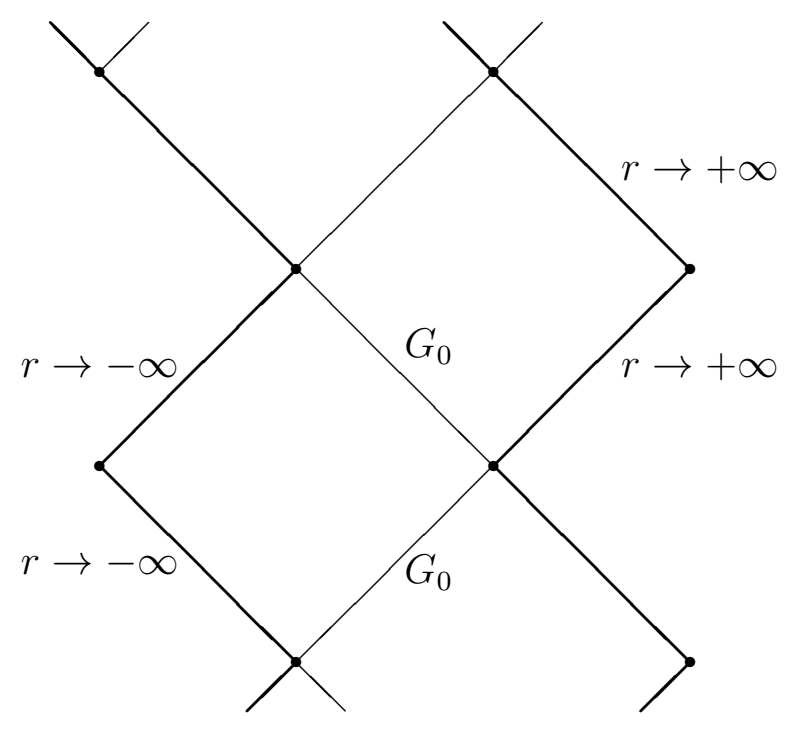

Figure 3: Penrose-diagram for the modified Sine-Gordon-soliton with $\kappa=2\left(G_{0}\right.$ denotes lines with $m r=0$ )

The soliton in these coordinates has the form

$$
\phi(r)=2 \arctan \sinh ^{-1}(m r)
$$


and the kink-number is $K=1$ as expected. Geodesics range from $r=-\infty$ to $r=+\infty$ for $E>\mu$ and oscillate around $r=0$ for $E<\mu$.

For $\kappa=4$ one obtains

$$
r(x)=-\frac{1}{2 m} \ln \left(1+e^{-2 m r}\right)
$$

and the metric in this case is

$$
d s^{2}=-\left(1-e^{2 m r}\right)^{2} d t^{2}+\frac{d r^{2}}{\left(1-e^{2 m r}\right)^{2}}
$$

Apart from $r=0(x \rightarrow \infty)$ another singularity arises at $r \rightarrow \infty$ which, after calculating the curvature

$$
R=8 m^{2} e^{2 m r}\left(1-2 e^{2 m r}\right)
$$

appears to be a true physical singularity. The space-time is now analogous to an extreme Reissner-Nordström-geometry [21]. The Penrose-diagram is shown in figure 4.

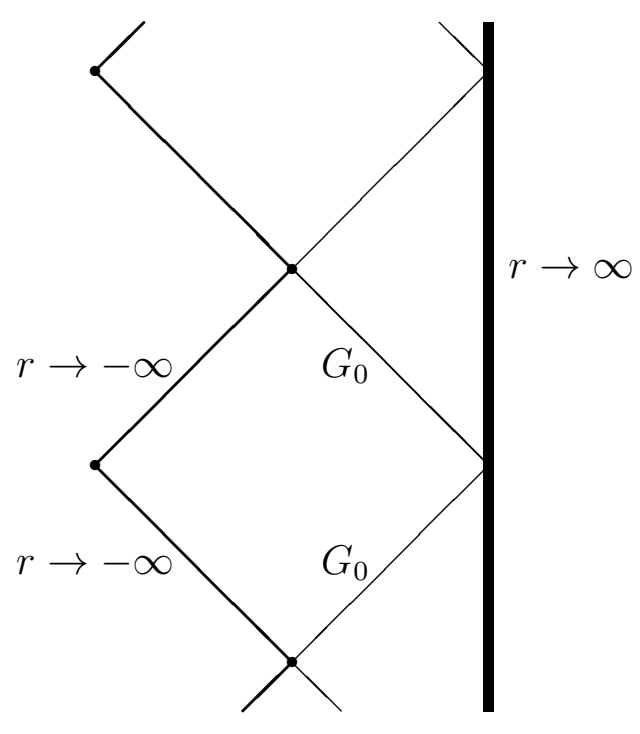

Figure 4: Penrose-diagram for the modified Sine-Gordon-soliton with $\kappa=4\left(G_{0}\right.$ denotes lines with $m r=0$; bold line represent the physical singularity)

The soliton is given by

$$
\phi(r)=2 \cot ^{-1} \sqrt{e^{-2 m r}-1}
$$


which is not defined in the region $r>0$. Therefore the soliton is not completed in this case. The missing half of it is cut off by the space-time singularity at $r \rightarrow \infty$.

Geodesics range from $r=-\infty$ to $r_{\max }=\frac{1}{2 m} \ln \left(1+\frac{E}{\mu}\right)$ for $E \geq \mu$ and oscillate around $r=0$ for $E<\mu$. The space-time singularity is unreachable for massive testparticles, but observable by light-rays.

\section{Conclusion}

The purpose of this work was to examine some aspects of two-dimensional gravity coupled to matter-fields with a special emphasize on the Sine-Gordon-model. There is no unique model for two-dimensional gravity and I used the one proposed by Mann, et al., because it seems to be the most natural analogue to general relativity in four dimensions. General properties of solitonic solutions in this model were discussed and different analytic solutions were found. The examination of the appropriate geometries showed very interesting and unexpected space-time-structures for these solutions.

Although I was not able to find other analytic solutions than the ones discussed in the above sections, this, of course, does not mean that there are no other solutions. It would be especially interesting to find soliton-solutions with a black hole metric, because a great part of the work done in two-dimensional gravity is connected with questions of black hole physics, like for example Hawking-radiation.

The Sine-Gordon-model was the subject of many investigations concerning quantum field theory in flat space-time and I hope that the solutions discussed in the present work may be useful starting points for similar investigations in quantum gravity.

After finishing my work I became aware of a recent preprint of Shin and Soh 22 dealing also with Sine-Gordon-solitons in two-dimensional gravity. They took the gravity-model of Callan, et al., and were able to discover a soliton-solution with a black hole metric, because the matter-gravity coupling they used is slightly different from the one I used in my work.

\section{Acknowledgments}

I wish to thank my wife Simone Stoetzel for type-setting the manuscript. 


\section{Appendices}

\section{A Antigravitational soliton}

It was mentioned in section 4 that the action (26) with negative values of $\kappa$ gives rise to an antigravitational model. In this appendix I give an example of such a solitonsolution for the pure Sine-Gordon-potential with vanishing cosmological constant. The same methods used in section 6 reveal the following solution for $\kappa=-4$ :

$$
\phi(x)=2 \arctan e^{m x}
$$

Actually, this is just one half of the kink, because it does not connect two successive minima (vacua) of the potential, but a minimum and an extremum. As in the last example of section 5 one might again expect an extension of space-time to complete the kink or a physical singularity to cut off the second half of the soliton.

The induced metric is

$$
d s^{2}=-\left(1+e^{2 m x}\right)^{2} d t^{2}+d x^{2}
$$

Note that this is in accordance with the metric solution (71) for the modified SineGordon-potential for $\alpha=0$, as it has to be. Introduce now a null-coordinate

$$
x^{*}=-\frac{1}{2 m} \ln \left(1+e^{-2 m x}\right)
$$

which gives the conformally flat metric

$$
d s^{2}=-\left(1-e^{2 m x^{*}}\right)^{-2}\left(d t^{2}-d x^{* 2}\right)
$$

The original domain of $x$ is mapped on the half-line $(-\infty, 0]$ and the space-time may be extended into the region $x^{*}>0$. The curvature is

$$
R=-8 m^{2} e^{2 m x^{*}}
$$

thus revealing a physical singularity at $x^{*} \rightarrow \infty$ and a coordinate singularity at $x^{*}=0$ (resp. $x \rightarrow \infty$ ). The Penrose-diagram for this case is shown in figure 5 .

The soliton in the new coordinate is

$$
\phi\left(x^{*}\right)=2 \cot ^{-1} \sqrt{e^{-2 m x^{*}}-1}
$$

which is not defined in the region $x^{*}>0$. Again the missing half of the kink is cut off by the singularity. 


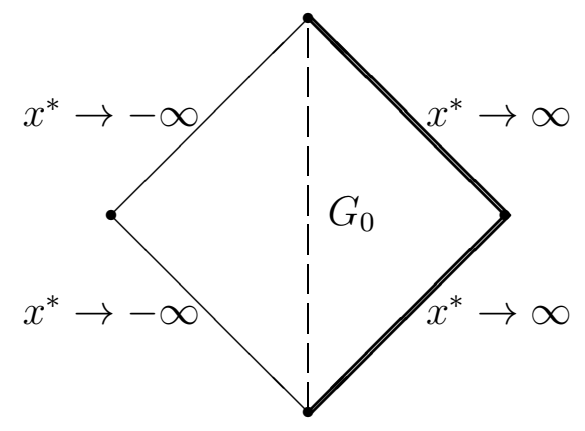

Figure 5: Penrose-diagram for antigravitational soliton in the Sine-Gordon-model with vanishing cosmological constant. $\left(G_{0}\right.$ denotes line with $x^{*}=0$; bold lines represent the physical singularity)

The geodesic equation is

$$
\left(\frac{d x^{*}}{d \lambda}\right)^{2}=\left[E^{2}\left(1-e^{2 m x^{*}}\right)^{2}-\mu^{2}\right]\left(1-e^{2 m x^{*}}\right)^{2}
$$

Massive testparticles stay always in one of the two halves of space-time depending on their initial conditions. Especially the point $x^{*}=0$ (which may be interpreted as the center of the soliton) can never be reached for $\mu \neq 0$. This shows the antigravitational character of the solution. The soliton repels every massive object.

\section{B Gravitationally bound soliton}

In this appendix I discuss a solution of the equations $(35,36)$ which can be interpreted as a gravitationally bound soliton. For a massless scalar-field the potential vanishes and equation (36) is easily solved by

$$
\theta(x)=\lambda \tanh (\lambda x)
$$

with the parameter $\lambda=\sqrt{\frac{\Lambda}{2}}$. This is a real solution for positive cosmological constant. Equation (35) yields

$$
\phi(x)=\phi_{0} \arctan e^{\lambda x}
$$

with an arbitrary constant $\phi_{0}$. The appropriate metric is given by

$$
d s^{2}=-\cosh ^{2}(\lambda x) d t^{2}+d x^{2}
$$


This is the anti-de Sitter geometry [23]. The space-time consists of infinitely many patches all filled by one soliton of the form (103) and completely separated for massive objects, which oscillate around one kink. Nevertheless, the different patches are observable with light-rays, because null-geodesics may reach from one to another part of the space-time.

\section{Treatment of coordinate singularities}

The usual treatment of coordinate singularities is patterned after the method of Kruskal introduced in dealing with the Schwarzschild-metric in four dimensions [24]. In two dimensions the recipe is the following: starting with the Schwarzschild-type metric, like for example (78), transform to a conformally flat coordinate system in such a way that the singularities disappear.

A generic Schwarzschild-type metric is

$$
d s^{2}=-F^{2}(r) d t^{2}+\frac{d r^{2}}{F^{2}(r)}
$$

A transformation to a conformally flat system is obviously achieved by

$$
r^{*}=\int \frac{d r}{F(r)}
$$

The resulting metric is

$$
d s^{2}=-F^{* 2}\left(r^{*}\right)\left(d t^{2}-d r^{* 2}\right)
$$

The singularity is at some point $r^{*}=r_{0}^{*}$. Find now a transformation to get rid of it:

$$
U=G\left(t, r^{*}\right), \quad V=H\left(t, r^{*}\right)
$$

The condition that the metric stays conformally flat yields conditions for the functions $H$ and $G$ :

$$
\dot{H}=G^{\prime} ; \quad H^{\prime}=\dot{G}
$$

with the general solutions

$$
\begin{aligned}
& G\left(t, r^{*}\right)=g\left(r^{*}+t\right)+h\left(r^{*}-t\right) \\
& H\left(t, r^{*}\right)=g\left(r^{*}+t\right)-h\left(r^{*}-t\right)
\end{aligned}
$$

and the new metric is

$$
d s^{2}=\frac{F^{* 2}\left(r^{*}\right)}{4 g^{\prime}\left(r^{*}+t\right) h^{\prime}\left(r^{*}-t\right)}\left(d U^{2}-d V^{2}\right)
$$

Therefore, the function $\Omega\left(r^{*}, t\right)=g^{\prime}\left(r^{*}+t\right) h^{\prime}\left(r^{*}-t\right)$ has to cancel the singularity in $F^{*}\left(r^{*}\right)$, which means

$$
\Omega\left(r^{*}, t\right) \stackrel{r^{*} \rightarrow r_{0}^{*}}{\longrightarrow} F^{* 2}\left(r_{0}^{*}\right), \quad t=\text { const } .
$$


For finite $r_{0}^{*}$ this is only possible if $\dot{\Omega}=0$ which is equivalent to $\Omega\left(r^{*}\right) \sim e^{\gamma r^{*}}$. An example for this is the antigravitational solution of appendix A, where $r_{0}^{*}=0\left(r^{*}\right.$ is called $x^{*}$ in that section), but near that value the conformal factor is

$$
F^{* 2}\left(r^{*}\right) \stackrel{r^{*} \rightarrow 0}{\longrightarrow} \frac{1}{\left(2 m r^{*}\right)^{2}}
$$

Therefore, this singularity cannot be removed by any coordinate transformation of the required type.

In all other examples of this paper $r_{0}^{*}$ is infinite. In that case, one has not to impose the additional condition $\dot{\Omega}=0$ and there is some more freedom than in the previous case. Nevertheless, the behavior of all these metrics near the singularity is of the form

$$
d s^{2} \sim \frac{-d t^{2}+d r^{* 2}}{4 m^{2} r^{* 2}} ; \quad r^{*} \rightarrow \infty
$$

Thus, one needs functions $g$ and $h$ such that

$$
g^{\prime}\left(r^{*}+t\right) h^{\prime}\left(r^{*}-t\right) \sim \frac{1}{r^{* 2}}
$$

for $r^{*} \rightarrow \infty$ and $t=$ const. or, in the same limit,

$$
\begin{aligned}
g^{\prime}\left(r^{*}+t\right) & \sim \frac{1}{\left(r^{*}+t\right)^{\alpha}} \\
h^{\prime}\left(r^{*}-t\right) & \sim \frac{1}{\left(r^{*}-t\right)^{2-\alpha}}
\end{aligned}
$$

which means for the functions themselves $(\alpha \neq 1)$ :

$$
\begin{aligned}
g\left(r^{*}+t\right) & \sim \frac{1}{\left(r^{*}+t\right)^{\alpha-1}} \\
h\left(r^{*}-t\right) & \sim \frac{1}{\left(r^{*}-t\right)^{1-\alpha}}
\end{aligned}
$$

In terms of the new coordinates

$$
\begin{aligned}
& U \sim\left(r^{*}\right)^{1-\alpha}+\left(r^{*}\right)^{\alpha-1} \\
& V \sim\left(r^{*}\right)^{1-\alpha}-\left(r^{*}\right)^{\alpha-1}
\end{aligned}
$$

If $r^{*}$ approaches the singularity both, $U$ and $V$, diverge; hence, the new coordinatesystem does not cover the whole space-time described by the coordinates $r^{*}$ and $t$. The last possibility left is the choice $\alpha=1$ in (116). In that case, one obtains

$$
U \sim \ln \left(r^{*}\right) ; \quad V \sim \ln \left(r^{*}\right)
$$

and again $U$ and $V$ diverge at the singularity. In other words, it is not possible to find a globally singularity-free system, if the singularity is of the form (114). 


\section{References}

[1] P. Callas; Am. J. Phys. 45 (1977) 833

[2] J. D. Brown; Lower Dimensional Gravity (Singapore: World Scientific 1988)

[3] R. B. Mann, S. F. Ross; Class. Quant. Grav. 9 (1992) 2335

R. B. Mann; Report No. WATPHYS TH-91/06 (unpublished)

[4] E. Witten; Phys. Rev. D 44 (1991) 314

C. G. Callan, S. B. Giddings, J. A. Harvey, A. Strominger; Phys.Rev. D 45 (1992) R1005

R. B. Mann, A. Shiekh, L. Tarasov; Nucl. Phys. B 341 (1990)134

R. B. Mann, M. S. Morris, S. F. Ross; Class. Quant. Grav 10(1993) 1477

R. B. Mann, S. M. Morsink, A. E. Sikkema, T. G. Steele; Phys. Rev.D 43 (1991) 3948

J. P. S. Lemos, P. M. Sá; Mod. Phys. Lett. A 9 (1994)771

J. P. S. Lemos, P. M. Sá; Phys. Rev. D49 (1994) 2897

J. P. S. Lemos; Class.Quant.Grav.12 (1995) 1081

R. Balbinot, P. R. Brady; Class. Quant. Grav 11 (1994) 1763

J. Y. Kim, H. W. Lee, Y. S. Myung; Phys. Lett. B 328 (1994) 291

A. Kumar, K. Ray; Preprint IP/BBSR/94-54, hep-th/9410068 (1994)

R. C. Myers; Phys.Rev. D50 (1994) 6412

[5] J. S. F. Chan, R. B. Mann; Class. Quant. Grav. 12 (1995) 351

A. E. Sikkema, R. B. Mann; Class. Quant. Grav. 8 (1991) 219

[6] J. Ambjørn, K. Ghoroku; Int. J. Mod. Phys. A 9 (1994) 5689

N. Sanchez; Nucl. Phys. B 266 (1986) 487

R. Floreanini; Ann. Phys. 167 (1986) 317

K. G. Akdeniz, Ö. F. Dayi, A. Kizilersü; Mod. Phys. Lett. A 7 (1992) 1757

S. W. Hawking, J. D. Hayward; Phys. Rev. D 49 (1994) 5252

Y. Kazama, Y. Satoh, A. Tsuchiya; Phys. Rev. D 51 (1995) 4265

[7] R. Jackiw; in Quantum Theory of Gravity, ed. S. Christensen (Bristol: Adam Hilger 1984) p. 403

R. Jackiw; Nucl. Phys. B 252 (1985) 343

[8] C. Teitelboim; in Quantum Theory of Gravity, ed. S. Christensen (Bristol: Adam Hilger 1984) p. 327

[9] R. B. Mann, S. M. Morsink, A. E. Sikkema, T. G. Steele; (see ref. 4)

[10] C. G. Callan, S. B. Giddings, J. A. Harvey, A. Strominger; (see ref. 4)

[11] J. P. S. Lemos, P. M. Sá; Phys. Rev. (see ref. 4) 
[12] R. Rajaraman; Solitons and Instantons (Amsterdam: North Holland 1982)

[13] R. Jackiw; Rev. Mod. Phys. 49 (1977) 681

C. Rebbi, G. Soliani; Solitons and Particles (Singapore: World Scientific 1984)

[14] M. Heusler, N. Straumann, Z. Zhou; Helv. Phys. Acta 66 (1993) 614

M. Heusler, S. Droz, N. Straumann; Phys. Lett. B 285 (1992) 21

M. Heusler, S. Droz, N. Straumann; Phys. Lett. B 271 (1991) 61

S. Droz, M. Heusler, N. Straumann; Phys. Lett. B 268 (1991) 371

[15] Ch. W. Misner, K. S. Thorne, J. A. Wheeler; Gravitation (San Francisco: W. H. Freeman and Company 1973)

[16] J. P. Lemos, P. M. Sá; Class. Quant. Grav. 11 (1994) L11

[17] R. B. Mann, S. F. Ross; Class. Quant. Grav. 10 (1993) 1405

[18] S. Coleman; Phys. Rev. D 11 (1975) 2088

[19] J. Ambjørn, K. Ghoroku; (see ref. 6)

[20] B. Carter; Phys. Rev. 141 (1966) 1242

[21] B. Carter; Phys. Lett. 21 (1966) 423

[22] H.-S. Shin, K.-S. Soh; Preprint hep-th/9501045 (1995) (to appear in Phys. Rev. D)

[23] M. Bañados, M. Henneaux, C. Teitelboim, J. Zanelli; Phys. Rev. D 48 (1993) 1506

[24] J. C. Graves, D. R. Brill; Phys. Rev. 120 (1960) 1506 